\title{
To compare the efficacy and safety of nifedipine sustained release with Ginkgo biloba extract to treat patients with primary Raynaud's phenomenon in South Korea; Korean Raynaud study (KOARA study)
}

\author{
Whan-Seok Choi $\cdot$ Chang-Jin Choi $\cdot$ Kyung-Soo Kim • \\ Jae-Ho Lee $\cdot$ Chan-Hee Song $\cdot$ Ju-Hye Chung • \\ Sun-Myeoung Ock $\cdot$ Jung-Bok Lee $\cdot$ Chul-Min Kim
}

Received: 18 March 2008 /Revised: 24 December 2008 / Accepted: 27 December 2008 / Published online: 22 January 2009

(C) The Author(s) 2009. This article is published with open access at Springerlink.com

\begin{abstract}
This study examined the efficacy and safety of nifedipine sustained release (nifedipine SR) compared with Ginkgo biloba extract as treatment for primary Raynaud's phenomenon (RP) in Korea. Primary RP were screened and assigned to either the nifedipine SR group (Group N) or the Ginkgo biloba extract group (Group G) in the ratio of 2:1. After a run-in period of 2 weeks, patients received treatment for 8 weeks. We observed the percent improvement of the RP attack rate between before and after the 8week treatment. Ninety-three subjects were randomly assigned. The percent improvement in Group $\mathrm{N}$ was $50.1 \%$ at 8 weeks after treatment, while it was $31.0 \%$ in Group $\mathrm{G}(p=0.03)$. No serious adverse events occurred, and almost adverse events were mild and improved without specific treatment. nifedipine SR was more effective than Ginkgo biloba extract for treatment of primary RP in Korean patients. Both drugs were tolerable with primary RP patients.
\end{abstract}

Keywords Ginkgo $\cdot$ Ginkgo biloba extract .

Nifedipine $\cdot$ Nifedipine sustained release .

Primary Raynaud's phenomenon .

Raynaud's phenomenon

W.-S. Choi $\cdot$ C.-J. Choi $\cdot$ K.-S. Kim • J.-H. Lee $\cdot$ C.-H. Song

J.-H. Chung $\cdot$ S.-M. Ock $\cdot$ C.-M. Kim $(\bowtie)$

Department of Family Medicine, Catholic University,

Seoul, Korea

e-mail: musofm@catholic.ac.kr

J.-B. Lee

Institute of Human Genomic Study, Korea University,

Seoul, Korea

\section{Introduction}

Raynaud's phenomenon (RP) was named after Dr. Maurice Raynaud, who found a condition in which people exposed to cold or emotional stress experienced clearly distinguished skin-color changes in their hands in 1862 [1]. This phenomenon, in its early stage, involves a condition that makes hands become white or blue due to excessive vasoconstriction when people are exposed to cold or emotional stress. Subsequently, their finger color changes to red by vasodilation, which is accompanied with itching or pain. RP occurs in two forms: secondary Raynaud's phenomenon where there is an associated disorder like a connective tissue disease and primary RP where there is not [2].

The prevalence of RP varies by gender, country and exposure to workplace vibration. One large US cohort study found symptoms in $9.6 \%$ of women and $8.1 \%$ of men, of whom $81 \%$ had primary RP [3]. According to population based surveys of various ethnic groups, the prevalence of unusual sensitive to cold on fingers is $12 \%$, $\mathrm{RP}$ in the healthy population is $3 \%$ to $5 \%$ [3]. Also, the prevalence of RP is reported $9.5 \%$ in labor group [4], in women $15.6-30 \%[5,6]$, and $11-21 \%$ in general practice setting [7]; 70-89\% of RP is Primary Raynaud's phenomenon of unknown origin $[2,8]$. Thus, the exact prevalence of primary RP is unknown, but primary RP is a common clinical disorder, especially in general practice setting.

In South Korea, we demonstrated $43 \%$ patients with abnormal cold sensation on hands or feet had RP, and primary RP was $73 \%$ out of RP in our previous study [9]. 
Calcium channel blockers (CCBs) are the most widely used agents for pharmacological treatment of RP $[2,10]$. Among CCBs, only use of nifedipine was approved for treatment of the disease in South Korea. There have been many randomized controlled trials of treatment of primary and secondary RP. However, there has been no clinical study for primary RP in South Korean patients. Also, 83\% of primary RP patients in South Korea usually have taken Ginkgo biloba for treatment RP; otherwise, only 5\% patients have taken CCBs for RP [9]. Furthermore, many general practitioners in South Korea also have prescribed Ginkgo biloba for RP (52\%) [11].

Ginkgo biloba extract has been used in oriental medicine at least since the fourteenth century. It is reputed anecdotally to be potent in circulatory disorder such as intermittent claudication [12].

We examined the efficacy and safety of nifedipine sustained release (nifedipine SR) compared with Ginkgo biloba extract to treat patients with primary RP.

\section{Materials and methods}

This study was a multicenter, open randomized, flexible dose clinical trial. The subjects in this study were selected from the Catholic University affiliated to St. Mary's Hospitals in South Korea. This study was conducted from December to next March when was winter season in Korea. The average atmospheric temperatures were $-1.3^{\circ} \mathrm{C}$ in December, $-6.0^{\circ} \mathrm{C}$ in January, $-5.1{ }^{\circ} \mathrm{C}$ in February, and $-0.2^{\circ} \mathrm{C}$ in March. Primary RP patients were given a screening number at Visit 1 (2 weeks), and they were randomly assigned to either nifedipine SR group (Group N) or ginkgo biloba extract group (Group $\mathrm{G}$ ) in the ratio of 2:1 at Visit 2 (0 week).

To determine whether subjects had RP, they were asked the following questions: (1) Are your fingers unusually sensitive to cold? (2) Do your fingers change color when exposed to cold? (3) Do they turn white, blue, or both? Subjects who answered 'Yes' to all three questions were diagnosed as having RP [2, 3] if a subject had normal antinuclear antibody and normal erythrocyte sedimentation rate, then the subject was diagnosed and enrolled with primary RP [3].

Patients participated in this clinical trial for a total period of 10 weeks. The patients submitted a written informed consent during the first screening period. Also, they were checked for vital signs, received a physical examination, hematological tests, 12-lead ECG, and a urine test. Those patients who were checked for the Color Chart [13] during a Run-in Period of 2 weeks and also filled in $75 \%$ or more of a patient diary were randomly assigned to either Group $\mathrm{N}$ or Group $\mathrm{G}$ in the ratio of 2:1 (at visit 2, week 0), and went on to receive treatment for 8 weeks. The color charts were consisted of 12-point color scale and a series of photographs illustrating blanching and cyanosis, has been developed to assist in the diagnosis of RP in 1988. The color charts showed a sensitivity of $90 \%$ and a specificity of $100 \%$ [13].

Also, patient diaries were distributed to obtain accurate and objective information related RP. Patients checked the frequency of cold exposures, the frequency of RP attacks, and the severity of RP attacks at the diary. The severity of RP ranged across a continuum of value but cannot easily be directly measured. So, we used 0-10 Visual Analog Scale (VAS) as a measurement instrument to determine the severity of RP attacks.

The patients had first taken for 1 week initial usual doses, nifedipine SR $30 \mathrm{mg}$ once daily or one tablet of Ginkgo biloba extract $40 \mathrm{mg}$ three times daily. The dose of both medicines was increased; to take two tablets each time of nifedipine SR $30 \mathrm{mg}$ once daily, or two tablets each time of Ginkgo biloba extract $40 \mathrm{mg}$ three times daily at visit three (1 week). However, if patient had experiences an adverse event for the past week, those patients were directed to take nifedipine SR $20 \mathrm{mg}$ once daily or to continue taking Ginkgo biloba extract $40 \mathrm{mg}$ three times daily. After one more week (visit 4, 2 weeks), their compliance with the drug was evaluated. If an adverse event was found, the dose was adjusted in order to enable the subjects to continue to take the medication for the remaining treatment period. When the whole therapeutic process was finished after 8 weeks of treatment, the efficacy and safety of each treatment were evaluated at visit 5 ( 8 weeks). Patients were telephoned three times during study to evaluate the safety and to improve patients' compliance with the study. Patients also submitted a patient diary at Visits 2, 3, 4, 5, and a premature discontinuation visit. The World Health Organization Quality of Life (WHO-QOL) questionnaire was evaluated at Visits 2 and 5 [14, 15]. Measurement of vital signs and a physical examination were conducted at every visit, and an Overall Assessment Question was evaluated. If a patient's compliance was under $70 \%$ throughout the whole treatment process of 8 weeks, the patient was dropped out of this study. The flow and procedure of this study are as shown in Fig. 1.

The primary end point in this study was the percent improvement $\left(R_{\text {treat }}\right)$ of rate of the attack frequency after cold exposures using color chart and patient diary.

$R_{\text {treat }}=\left(R_{\text {baseline }}-R_{\text {week } 8}\right) / R_{\text {week } 8} \times 100(\%)$

$R_{\text {baseline }}$ and $R_{\text {week } 8}$ meant the real RP attack rate to the frequency of exposure that could be caused RP attack at baseline and week 8 visits. We also evaluated WHO-QOL, 


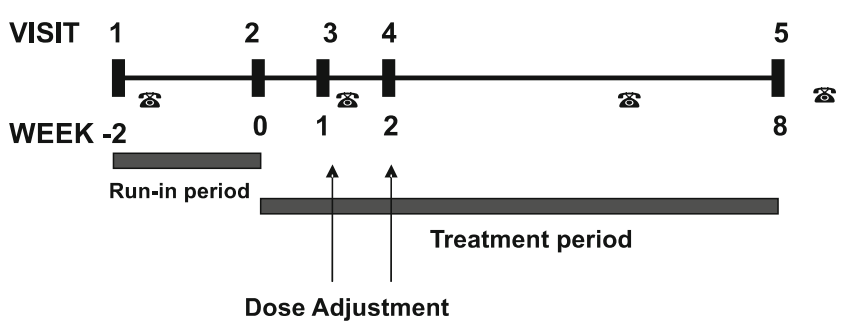

Fig. 1 Study flow of KOARA Study (after 2 weeks run-in period, patients randomly assigned and received the study medications. Patients were followed up for dose adjustment two times with 1-week interval. Patients had taken the medication for 8 weeks in total. During whole trial period, patients were called four times to evaluate the safety and to improve their compliance with study (telephone symbol), KOARA study; Korean Raynaud study)

improvement rate of attack frequency compared to baseline by time, and safety profiles as secondary end points.

To calculate sample size for this study, we assumed that difference of the percent change of the attack between two groups was $20 \%$ and considered standard deviation of the percent change of the attack in each group was 52. Based on these estimates, the required sample size was 60:30 in each group with a power of $80 \%$ and an $\alpha$ of 0.05 .

To compare general characteristics and baseline homogeneity of disease status between two groups, chi-squared test and Fisher's exact test were used for discrete variables, and Wilcoxon's rank sum test and two-sample $t$ test were performed for continuous variables. Efficacy variables were compared with two-sample $t$ test and performed chi squared test to compare overall satisfaction between two groups. Repeated ANOVA was performed to evaluate blood pressure changes over three visits between groups. SAS 8.2 program was used for statistical analysis.

Research process, execution, evaluation, and record of this clinical study were designed so that the we could execute this clinical trial under the terms of Good Clinical Practice and the principles of the Declaration of Helsinki, and we were approved by the Independent Ethics Committee and the Investigational Review Board for the written informed consent and other written information as provided to patients.

In this study, the intention-to-treat (ITT) group is defined as subjects who met all the qualifications and were administered the test drug at least one time. If missing data occurred due to dropout, it was corrected by Last Observation Carried Forward. Results are basically reported for the ITT group. Additionally, we intended to conduct a clinical trial for a Per-Protocol (PP) group and provide the results, and if there was a difference in analysis result between ITT group and PP group, a cause would be searched for. SAS 8.2 program was used for statistical analysis.

\section{Results}

Characteristics of subjects

Out of the 132 patients screened, 39 subjects were dropped out during the screening period. Finally, 93 subjects were randomized. All randomized patients received study drugs: 61 patients received nifedipine SR (Group N) and 32 patients received ginkgo biloba extract (Group G). Sixtyfour subjects were completed (Group N: 42, Group G: 22) this clinical trial during the 8 weeks (Fig. 2).

The randomized subjects were $26 \%$ male and $74 \%$ female and had a mean age 39.2 years. Almost all patients were nonsmoker $(84 \%)$. At baseline, mean blood pressure for each Group N and Group G was 118.2/73.4 and 118.4/ $73.8 \mathrm{mmHg}$. Pulse per minute and body mass index were also similar in both groups (Table 1).

The mean age of the first time when patients experiences primary RP was before 16.5 years for Group $\mathrm{N}$ and before 13.9 years for Group G. 33\% of Group N subjects had the experience of primary RP treatment, $28 \%$ for Group G. $42 \%$ of Group N subjects had the 1st degree family history of primary RP, while $10 \%$ subjects had the family history for Group G. All subjects of both groups answered that the hands color changed mostly when they went into the cold outside. The answers were followed in order; when they soaked their hands in cold water $(83 \%)$, touched cold materials $(69 \%)$, and were exposed to cold wind $(27 \%)$ for example, air-conditioning room.

The mean number of exposures for a week was 18.9 times in Group N and 18.4 times in Group G during the 2-week screening period. During this time, the number of color changes (RP attacks) within each group was 14.3 and 13.9, so the mean attack rate during the run-in period was $73.7 \%$ in group $\mathrm{N}$ and $73.4 \%$ group $\mathrm{G}$. The mean intensity at exposure in each group was 5.07 and 5.05 using a VAS to measure the intensity on scale of 10. No statistical significant differences in the number of exposures, the number of color changes, the attack rate, or pain intensity was found between the two groups.

Primary end point efficacy

As a result of comparing the attack rate at the baseline compared to 8 weeks after treatment in the ITT group, the percent change was $50.1 \%$ in Group N, while it was $31.0 \%$ in Group G. That is, about $50 \%$ decrease was found in the attack rate at the screening period compared to the attack rate after treatment in the Group $\mathrm{N}$, which implies there is a statistically significant difference between the two groups (Table 2). No difference in average attack severity was found between the two groups. A similar analysis result was shown 
Fig. 2 Classification of KOARA Study participants ( $F / U$ follow up)

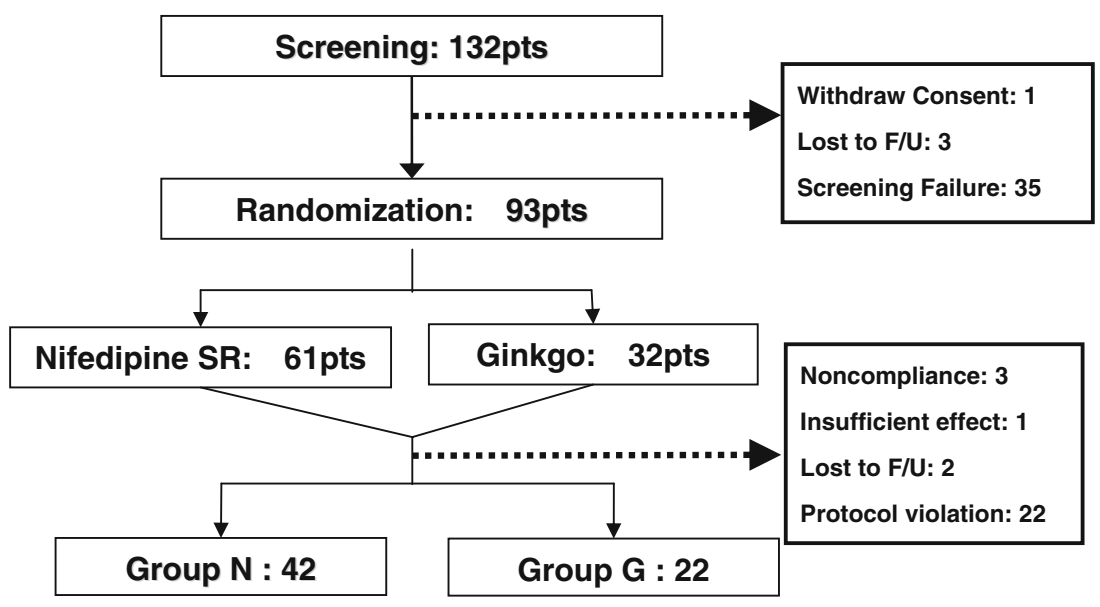

between PP group and ITT group (Group N; 61.3\%, Group G; 38.4\% improved, $p=0.037$ ).

Secondary end points

The percent change of the attack rate at 8 weeks after treatment compared to the baseline shows an increase from $11.5 \%$ when the treatment was started in Group $\mathrm{N}$ to $50.1 \%$ at the period of treatment was finished, and the most significant change was shown at 2 weeks after treatment was finished. In Group $\mathrm{G}$, the attack rate was increased from $11.4 \%$ at 1 week after treatment was started, stayed at about $20 \%$ until 3 week after treatment, and then increased by $31.0 \%$ at 2 weeks after treatment was finished. However, no statistical significance in percent change of the attack rate by point of time was shown between the two groups ( $p=$ 0.0920; Fig. 3).

There were significant differences in overall satisfaction for treatment from baseline for both drugs. However, Group $\mathrm{N}$ produced significantly greater satisfactions at visit $4(p=$ $0.04)$ and visit $5(p=0.001)$.There was no significant difference or change in WHO-QOL [14, 15] for both medications.

Safety profiles

No serious adverse events or abnormal drug reactions occurred in this study. Total subjects of experiencing drugrelated adverse events was $16(26.2 \%)$ for Group $\mathrm{N}$ and seven $(21.9 \%)$ for Group $\mathrm{G}$, and the total number of drug relevant adverse events was 28 in Group $\mathrm{N}$ and ten in

Table 1 General characteristics of subjects

\begin{tabular}{|c|c|c|c|c|c|}
\hline & & Group $\mathrm{N}(n=61)$ & Group G $(n=32)$ & Total $(n=93)$ & $p$ Value $^{\mathrm{a}}$ \\
\hline \multirow[t]{2}{*}{ Sex } & Male & $12(19.7 \%)$ & $12(37.5 \%)$ & $24(25.8 \%)$ & \multirow[t]{2}{*}{$0.0620^{\mathrm{b}}$} \\
\hline & Female & $49(80.3 \%)$ & $20(62.5 \%)$ & $69(74.2 \%)$ & \\
\hline Age (years) & & $37.67 \pm 12.27$ & $42.13 \pm 14.41$ & $39.20 \pm 13.14$ & $0.1528^{\mathrm{c}}$ \\
\hline \multirow[t]{3}{*}{ Smoking $(N=92)$} & Non-smoker & $52(86.7 \%)$ & $25(78.1 \%)$ & $77(83.7 \%)$ & \multirow[t]{3}{*}{$0.0992^{\mathrm{d}}$} \\
\hline & Ex-smoler & $1(1.7 \%)$ & $4(12.5 \%)$ & $5(5.4 \%)$ & \\
\hline & Current smoker & $7(11.7 \%)$ & $3(9.4 \%)$ & $10(10.9 \%)$ & \\
\hline \multirow[t]{2}{*}{ Occupation } & Blue colar & $14(23.0 \%)$ & $8(25.0 \%)$ & $22(23.7 \%)$ & \multirow[t]{2}{*}{0.8252} \\
\hline & White colar & $47(77.1 \%)$ & $24(75.0 \%)$ & $71(76.3 \%)$ & \\
\hline \multirow[t]{2}{*}{ Systolic BP (mmHg) } & Visit 1 & $118.20 \pm 19.47$ & $118.41 \pm 16.85$ & & 0.9573 \\
\hline & Visit 2 & $115.15 \pm 16.19$ & $116.91 \pm 13.84$ & & 0.6028 \\
\hline \multirow[t]{2}{*}{ Diastolic BP (mmHg) } & Visit 1 & $73.82 \pm 11.77$ & $75.31 \pm 8.48$ & & 0.7667 \\
\hline & Visit 2 & $72.93 \pm 10.42$ & $72.28 \pm 9.32$ & & 0.2451 \\
\hline \multirow[t]{2}{*}{ Pulse (times/min) } & Visit 1 & $68.00 \pm 7.61$ & $67.22 \pm 10.94$ & & 0.6880 \\
\hline & Visit 2 & $66.13 \pm 6.49$ & $67.28 \pm 8.89$ & & 0.5209 \\
\hline BMI $\left(\mathrm{kg} / \mathrm{m}^{2}\right)$ & & $22.35 \pm 6.58$ & $21.79 \pm 2.73$ & & 0.5675 \\
\hline
\end{tabular}

$B P$ blood pressure, $B M I$ body mass index

${ }^{\text {a }}$ Chi-square test

${ }^{\mathrm{b}}$ Wilcoxon's rank sum test

${ }^{c} p$ Value of sex corresponded to testing significant difference of gender distribution (male or female) between two groups

${ }^{d}$ Fisher's Exact Test 
Table 2 Primary endpoint in intention-to-treat (ITT) group

\begin{tabular}{llll}
\hline & & Treatment & \\
\cline { 3 - 4 } & & Group N $(n=61)$ & $73.9 \pm 27.0$ \\
Frequency of attack (\%) & $R_{\text {baseline }}$ & $73.7 \pm 24.2$ & $54.5 \pm 45.9$ \\
Percent improvement of primary RP attack rate (\%) & $R_{\text {week8 }}$ (after treatment) & $40.7 \pm 42.4$ & $31.0 \pm 45.9 *$ \\
\hline
\end{tabular}

ITT analysis was performed for patients who have taken at least one dose of study medication $R P$ Raynaud's phenomenon $* p=0.038$

Group G. The severity of adverse events was mostly mild (Group $\mathrm{N}=92.9 \%$, Group $\mathrm{G}=80.0 \%$ ). Those adverse events were improved without specific treatment. The most commonly reported adverse event was headache from both groups, and followed facial flushing, gastrointestinal troubles, edema, and dizziness from total ITT groups (Table 3).

There was no significant difference between the two groups in systolic or diastolic blood pressures before and after treatment. Both blood pressure and pulse rate were remained steady in both groups compared to the levels recorded during the screening period.

\section{Discussion}

Although some clinical trials on patients with primary or secondary RP using nifedipine have been performed intermittently [16-19], this study was the first clinical trial using South Korean patients with primary RP as its subjects.

Previous studies have evaluated the efficacy of medicines for primary RP simply on the basis of the frequency of attacks without taking into account the diverse changes in season or lifestyles. While in our study, the primary end point was set to the level of improvement in attack rate,

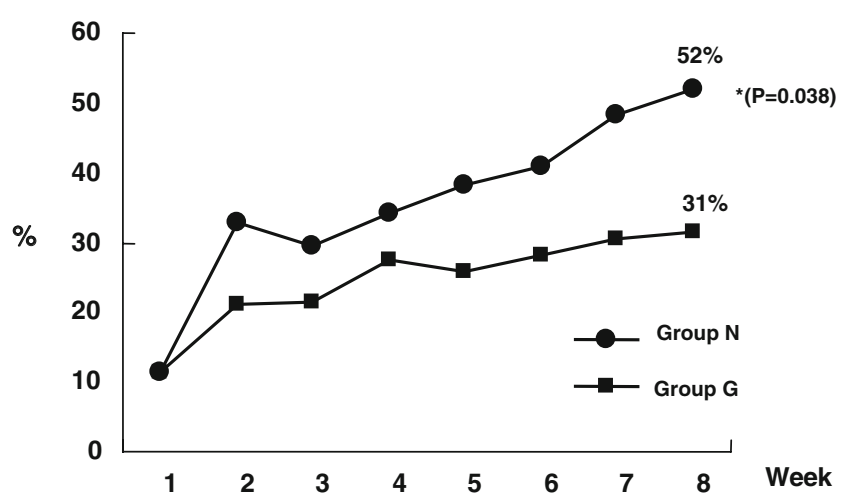

Fig. 3 Percent improvement of primary RP attack rate by time (weeks) in KOARA ITT subjects because the frequency of exposure that the RP attack can develop may be affected by a variety of factors including seasonal variations and personal lifestyles [20]. In addition, we used color chart for the purpose of more accurately patients' RP attack assessment [13].

The average age was 39 years and the percent of female patients was $74 \%$ and that of male patients was $26 \%$. This subject pool is consistent with the report that the relevant symptoms of primary RP develop primarily at the age range of 20 to 40 years, and the disease is prevalent in the female population rather than male [21]. The average age of early onset, which in this study was found to be 15.5, is also consistent with reported findings in other studies [22]. Cases with family history accounted for $30 \%$ on average, which is also consistent with findings in other studies [23]. While other studies considered exposure to coldness or stress as attack conditions or factors [3], this study considered more concrete conditions or factors such as when patients went out into the cold air, handled a cold object, were exposed to operating an air conditioner, entered a cold room, or were exposed to excessive stress, on the basis of the data obtained from the preliminary investigation.

In evidence-based medicine, the therapeutic principles for primary RP should involve lifestyle modification and medication with nifedipine [2, 3, 24]. Nevertheless, in South Korea, herb medicines, synthetic nutrients, or bloodcirculation-improving agents have been often administered to patients with primary RP without proven evidence [9]. Also, we previously demonstrated that many Korean primary practitioners prescribe blood-circulation-improving agents like Ginkgo biloba extract rather than other drugs to patients with primary RP [11].

As a result of this study that treated Korean patients with primary RP, nifedipine SR showed a 50\% improvement in attack rate, and a Ginkgo biloba extract preparation showed a 30\% improvement. The difference between the two groups was statistically significant, suggesting that the nifedipine SR has relatively dominant efficacy. Prescribed by most physicians, Ginkgo biloba extract is less effective than nifedipine SR. Though, because our study was absent 
Table 3 Summary of safety profiles in ITT Group

\begin{tabular}{|c|c|c|c|}
\hline & Group $\mathrm{N}(n=61)$ & Group G $(n=32)$ & $p$ Value $^{\mathrm{a}}$ \\
\hline Subjects who experienced drug-relevant adverse events & $16(26.2 \%)$ & $7(21.9 \%)$ & $0.6438^{\mathrm{b}}$ \\
\hline Serious abnormal reaction & 0 & 0 & \\
\hline Drug-relevant adverse events & 28 & 10 & \\
\hline \multicolumn{4}{|l|}{ Type of adverse events } \\
\hline Headache & $11(18.0 \%)$ & $3(9.4 \%)$ & 0.3860 \\
\hline Facial flushing & $6(9.8 \%)$ & $0(0 \%)$ & 0.0901 \\
\hline GI troubles & $3(4.9 \%)$ & $3(9.4 \%)$ & 0.4107 \\
\hline Edema & $3(4.9 \%)$ & $3(9.4 \%)$ & 0.4107 \\
\hline Dizziness & $1(1.6 \%)$ & $1(3.1 \%)$ & 1.0000 \\
\hline Palpitation & $1(1.6 \%)$ & $0(0 \%)$ & 1.0000 \\
\hline Others minor events & $3(4.9 \%)$ & $0(0 \%)$ & 1.0000 \\
\hline Severity & & & 0.2788 \\
\hline Mild & $26(92.9 \%)$ & $8(80.0 \%)$ & \\
\hline Moderate & $2(3.1 \%)$ & $2(20.0 \%)$ & \\
\hline Severe & $0(0 \%)$ & $0(0 \%)$ & \\
\hline
\end{tabular}

${ }^{\text {a }}$ Fisher's Exact Test

${ }^{\mathrm{b}}$ Chi-square test

a placebo arm, we could not compare the efficacy between Ginkgo and placebo effect directly. However, considering the efficacies of placebo in some previous studies were similar the improvement rate of Ginkgo in our study, the efficacy of Ginkgo was not seemed high [25, 26].

Regarding the secondary end points, as a result of evaluating the level of improvement in attack rates over time, Group $\mathrm{N}$ began with $11.5 \%$ at the baseline and showed a $50.1 \%$ improvement during 2 weeks before completion. Group $\mathrm{G}$ began with $11.4 \%$ at the first treatment week and showed a $31.0 \%$ improvement during 2 weeks before completion. However, the difference between the two groups was not statistically significant $(p=0.0920)$. This lack of statistical significance may be attributed to the relative short period of treatment and small number of study populations so that if the treatment lasted for a longer time and had more study subjects, different outcomes from those mentioned above are expected.

With regard to the overall satisfaction with the outcomes after treatment, Groups N and G showed $89 \%$ and $59 \%$, respectively. The remarkable difference between them was identified.

However, WHO-QOL showed no difference between the two groups [14, 15]. The reason why there was no significant difference in the variable, even though primary RP has significant influence upon daily livings or activities, may be either the relatively short period of study or that WHO-QOL-Kor. is an inappropriate scale to evaluate the QOL among the Korean patients with RD [11, 15]. Therefore, it is considered that new tools to evaluate the QOL among the patients with primary RP should be researched and developed over time.
There was no problem concerning safety in both Groups $\mathrm{N}$ and $\mathrm{G}$. There was no case reported in relation to abnormal blood pressure in Group $\mathrm{N}$ including the patients with primary RP who had normal blood pressure and took $30-60 \mathrm{mg}$ of nifedipine SR.

Our study had several limitations. First, this study had the relative short period of treatment. So, we could not determine the long-term treatment effect in both groups. Second, some of our patients were dropped out of the study due to protocol violation. Third, because this study was the open trial, so the investigators could recognize at which group the patient was assigned from visit 3. This nonblinding method would influence the evaluation of the outcome. But, we tried to reduce this bias by using color chart for more accurate and objective measurement of RP attacks and the percent change improvement of attack rate as primary end point. Forth, although capillaroscopy is a nice additional criterion to define the presence of primary RP, we could not use it in this study. Despite these limitations, the result of this study provided useful information on the efficacy, safety, and patient satisfaction of both drugs in Korean primary RP patients.

In conclusion, as a result of comparing nifedipine SR to Ginkgo biloba extract as treatment for Korean patients with primary RP, nifedipine SR was more effective than Ginkgo biloba extract and both of the drugs were safe. Prescribed by most physicians, Ginkgo biloba extract is less effective than nifedipine SR.

Acknowledgments This study was supported by Bayer HealthCare Korea. 
Disclosure None

Open Access This article is distributed under the terms of the Creative Commons Attribution Noncommercial License which permits any noncommercial use, distribution, and reproduction in any medium, provided the original author(s) and source are credited.

\section{References}

1. Raynaud M (1864) On local asphyxia and symmetrical gangrene of extremities. Selected Monographs. New Sydenham Society, London, p 1888

2. Porter JM, Rivers SP, Anderson CJ, Baur GM (1981) Evaluation and management of patients with Raynaud's Syndrome. Am J Surg 142:183-89

3. Wigley FM (2002) Raynaud's phenomenon. N Engl J Med 347:1001-1008

4. Walker DD, Jones B, Ogston S, Tasker EG, Robinson AJ (1985) A study of white finger in the gas industry. Br J Ind Med 42:672677

5. Leppert J, Aberg H, Rinqvist I, Sorensson S (1987) Raynaud's phenomenon in a female population: prevalence and association with other conditions. Angiology 38:871-877

6. Keil JE, Maricq HR, Weinrich MC, McGregor AR, Diat F (1991) Demographic, Social and clinical correlates of Raynaud phenomenon. Int J Epidemiol 21:221-224

7. Silman A, Holligan S, Brennan P, Maddison P (1990) Prevalence of symptoms of Raynaud's phenomenon in general practice. BMJ 301:590-592

8. Riera G, Vilardell M, Vaque J, Fonollosa V, Bermejo B (1993) Prevalence of Raynaud's phenomenon in a healthy Spanish population. J Rheumatol 20:66-69

9. Choi WS, Ock SM, Byeon JH (2003) The Prevalence of Diagnosis as Raynaud's disease among the People complaint of abnormal sensation on hands and feet. J Korean Acad Fam Med 24:1085-1089

10. Thompson AE, Pope JE (2005) Calcium channel blockers for primary Raynaud's phenomenon: a meta-analysis. Rheumatol $44: 145-150$

11. Kim CM, Ock SM, Chung JH, Jang HS, Yoo DJ, Lee JB, Choi WS (2007) Recognition of family practitioners on cold hands/feet syndrome and Raynaud's disease. J Korean Acad Fam Med 28:339-345
12. Pittler MH, Ernst E (2000) Ginkgo biloba extract for the treatment of intermittent claudication: a meta-analysis of randomized trials. Am J Med 108:276-281

13. Maricq HR, Winrich MC (1988) Diagnosis of Raynaud's phenomenon assisted by color charts. J Rheumatol 15:454-459

14. WHOQOL-Group (1993) Study protocol for the World Health Organization project to develop a Quality of Life assessment instrument (WHOQOL). Qual Life Res 2:153-159

15. Min SK, Kim KI, Lee CI, Jung YC, Suh SY, Kim DK (2002) Development of the Korean versions of WHO Quality of Life scale and WHOQOL-BREF. Qual Life Res 11:593-600

16. Thompson SE, Shea B, Welch V, Fenlon D, Pope JE (2001) Calcium-channel blockers for Raynaud's phenomenon in systemic sclerosis. Arthritis Rheum 44:1841-1847

17. Rademaker M, Cooke ED, Almond NE (1989) Comparison of intravenous infusions of iloprost and oral nifedipine in treatment of Raynaud's phenomenon in patients with systemic sclerosis: a double blind randomized study. BMJ 298:561-564

18. Dziadzio M, Denton CP, Smith R, Howel K, Blann A, Bowers E (1999) Losartan therapy for Raynaud's phenomenon and scleroderma: clinical and biochemical findings in a fifteen-week. Randomized, parallel-group, controlled trial. Arthritis Rheum 42:2646-2655

19. Coleiro B, Marshall SE, Denton CP (2001) Treatment of Raynaud's phenomenon with selective serotonin reuptake inhibitor fluoxetine. Rheumatology 40:1038-1043

20. Watson HR, Robb R, Belcher JJ (1992) Seasonal variation of Raynaud's phenomenon secondary to systemic sclerosis. J Rheumatol 26:1734-1737

21. Block JA, Sequeira W (2001) Raynaud's phenomenon. Lancet 357:2042-2048

22. Creager MA, Dzau VJ (2001) Raynaud's phenomenon; Harrison's principles of internal medicine, 15th edn. McGraw-Hill, New York, pp 1438-1439

23. Freeman RR, Meyes MD (1996) Familial aggregation of primary Raynaud's disease. Arthritis Rheum 39:1189-1191

24. National Institutes of Health (1993) Facts about Raynaud's phenomenon. National Heart, Lung and Blood Institute, National Institute of Health, Public Health Service, US Department of Health and Human Services, Bethesda Publication No. 93-2263

25. Sarkozi J, Bookman A, Mahon W, Ransay C, Detsky AS, Keystone EC (1986) nifedipine in the treatment of idiopathic Raynaud's syndrome. J Rheumatol 13:331-336

26. Raynaud's Treatment Study Investigators (2000) Comparison of sustained-release nifedipine and temperature biofeedback for treatment of primary Raynaud phenomenon. Results from a randomized clinical trial with 1-year follow-up. Arch Intern Med 160:1101-1108 